\title{
SUBFIELDS OF ALGEBRAICALLY MAXIMAL KAPLANSKY FIELDS
}

\author{
F.-V. KUHLMANN
}

\begin{abstract}
Using the ramification theory of tame and Kaplansky fields, we show that maximal Kaplansky fields contain maximal immediate extensions of each of their subfields. Likewise, algebraically maximal Kaplansky fields contain maximal immediate algebraic extensions of each of their subfields. This study is inspired by problems that appear in henselian valued fields of rank higher than 1 when a Hensel root of a polynomial is approximated by the elements generated by a (transfinite) Newton algorithm.
\end{abstract}

\section{IntRoduCtion}

For real functions, the Newton Algorithm is a nice tool to approximate their zeros. An analogue works in valued fields, such as the fields of $p$-adic numbers. Take a complete discretely valued field $K$ with valuation ring $\mathcal{O}$ and a polynomial $f$ in one variable with coefficients in $\mathcal{O}$. If $b \in \mathcal{O}$ satisfies $v f(b)>2 v f^{\prime}(b)$, then set $x_{0}:=b$ and

$$
x_{i+1}:=x_{i}-\frac{f\left(x_{i}\right)}{f^{\prime}\left(x_{i}\right)}
$$

for $i \geq 0$. It can easily be shown, using the Taylor expansion of $f$, that this sequence is a Cauchy sequence, and if a limit exists, then it is a root of $f$. This fact can be used to prove Hensel's Lemma in the complete fields of $p$-adic numbers, which states the existence of $p$-adic roots of suitable polynomials.

Date: 21. 12. 2017.

2000 Mathematics Subject Classification. Primary 12J10; Secondary 12J25, 13A18, $12 \mathrm{Y} 05$.

Key words and phrases. valued field, Kaplansky field, tame field, Newton algorithm.

The author would like to thank Anna Blaszczok for the careful proofreading of an earlier version of this manuscript, and Pablo Cubides Kovacsics for helpful remarks.

The initial work for this paper was done while the author was a visiting professor at the Institute of Mathematics at the University of Szczecin; he would like to thank his colleagues at this Institute for their great hospitality and support. 
Hensel's Lemma also holds in other valued fields (such as power series fields with arbitrary ordered abelian groups of exponents), which can be much larger than the fields of $p$-adic numbers. Still, it can be proved using the Newton algorithm, but if the value group of the field is not archimedean ordered (i.e., if it is not an ordered subgroup of $\mathbb{R})$, then the algorithm may not deliver the root after the first $\omega$ many iterations and transfinite induction is needed. For those who do not like the technicalities of transfinite induction, S. Prieß-Crampe presented in 1990 an elegant alternative (see [8]). The setting of the Newton algorithm gives rise to a contracting function

$$
\Phi(x):=x-\frac{f(x)}{f^{\prime}(x)}
$$

defined on a suitable subset of the field, whose unique fixed point, if it exists, is a root of the polynomial. The existence is guaranteed if $f$ satisfies the assumptions of Hensel's Lemma and the valued field from which its coefficients are taken is spherically complete (which is a property shared by all power series fields). While it is not the original definition, it suffices here to say that a valued field is spherically complete if and only if every pseudo Cauchy sequence in the sense of [3] has a (pseudo) limit.

The members of the sequence obtained from the Newton algorithm are approximations to a root of $f$. If the value group of the field in which we are working is not archimedean ordered, then the problem can appear that the sequence is not Cauchy, but only a pseudo Cauchy sequence. The limits of pseudo Cauchy sequences which are not Cauchy are not uniquely determined. In a recent paper [9], Prieß-Crampe considers the question whether this situation can be repaired, for a given polynomial $f$, by passing to a suitable subfield of $K$ in which the sequence becomes Cauchy and has a limit. She proves that this can indeed be done when $K$ is a spherically complete Kaplansky field. Let us provide the necessary definitions and background.

We will work with (Krull) valuations $v$ and write them in the classical additive way, that is, the value group of $v$ on a field $K$, denoted by $v K$, is an additively written ordered abelian group, and the ultrametric triangle law reads as $v(a+b) \geq \min \{v a, v b\}$. We denote by $K v$ the residue field of $v$ on $K$, by $v a$ the value of an element $a \in K$, and by $a v$ its residue.

A polynomial $f$ over a field of characteristic $p>0$ is called additive if $f(a+b)=f(a)+f(b)$ for all elements $a, b$ in any extension of the field. This holds if and only if $f$ is of the form $\sum_{0 \leq i \leq n} c_{i} X^{p^{i}}$. Following 
I. Kaplansky, we call a polynomial $g$ a $p$-polynomial if $g=f+c$ where $f$ is an additive polynomial and $c$ is a constant.

A valued field $(K, v)$ is called a Kaplansky field if char $K v=0$ or if it satisfies Kaplansky's hypothesis A: for char $K v=p>0$,

(A1) every $p$-polynomial with coefficients in $K v$ has a zero in $K v$,

(A2) $v K$ is $p$-divisible.

Prieß-Crampe proves her main result in [9] by a direct application of this formulation of hypothesis A. While this proof is not without interest, a more modern approach can lead to more insight, as we will show in the present paper. A lot of important work has been done over time by several authors in order to better understand Kaplansky's hypothesis A, e.g. by G. Whaples in [11], by F. Delon in her thesis, and in the paper [7]. The final touch to this development was given by Kaplansky himself, in cooperation with D. Leep (see [4, Section 9]). We will use the following characterization. By Theorem 1 of [11] and the other cited sources, hypothesis $\mathrm{A}$ is equivalent to the conjunction of the following three conditions, where $p$ denotes the characteristic of the residue field:

(K1) if $p>0$, then the value group is $p$-divisible,

(K2) the residue field is perfect,

(K3) the residue field admits no finite separable extension of degree divisible by $p$.

A valued field is henselian if it satisfies Hensel's Lemma, or equivalently, admits a unique extension of its valuation to its algebraic closure. Note that every algebraic extension of a henselian field is again henselian, with respect to the unique extension of the valuation. A henselization of a valued field $(K, v)$ is an algebraic extension which is henselian and minimal in the sense that it can be embedded over $K$ in every other henselian extension field of $(K, v)$. Henselizations exist for every valued field $(K, v)$, in fact, every henselian extension field of $(K, v)$ contains a henselization of $(K, v)$. Henselizations are unique up to valuation preserving isomorphism over $K$. Therefore, we will speak of "the henselization of $(K, v)$ " and denote it by $\left(K^{h}, v\right)$.

An extension $(L \mid K, v)$ of valued fields is called immediate if the canonical embeddings of $v K$ in $v L$ and of $K v$ in $L v$ are onto, in other words, value group and residue field remain unchanged. A valued field is called maximal if it does not admit any nontrivial immediate extensions; by [3, Theorem 4], this holds if and only if it is spherically complete. A valued field is called algebraically maximal if it does not 
admit any nontrivial immediate algebraic extension. Since henselizations are immediate algebraic extensions, every algebraically maximal field is henselian.

The notion of "purely wild extension" (an algebraic extension of a henselian field that is linearly disjoint from all tame extensions) was introduced in [7]. We will give the precise definitions for both types of extensions in the Preliminaries Section.

The following is our main theorem:

Theorem 1.1. Take a valued field extension $(L \mid K, v)$, with $(L, v)$ an algebraically maximal Kaplansky field. Then $L$ contains a maximal immediate algebraic extension of $(K, v)$, as well as a maximal purely wild extension of the henselization of $(K, v)$ inside of $(L, v)$.

If $(L, v)$ is maximal, then it also contains a maximal immediate extension of $(K, v)$.

Modulo the fact that a valued field is maximal if and only if it is spherically complete, the last assertion of this theorem is proved in [9, Theorem 3.5]. As already indicated, we present a different proof, which will be based on the ramification theory of Kaplansky and tame fields. A crucial tool in this proof is the following analogue of Lemma 3.7 of [6], which deals with the case of tame fields. Interestingly, in the case of algebraically maximal Kaplansky fields we do not need the assumption of that lemma that the residue field extension $L v \mid K v$ be algebraic.

Proposition 1.2. Take an algebraically maximal Kaplansky field $(L, v)$ and let $K$ be a relatively algebraically closed subfield of $L$. Then also $(K, v)$ is an algebraically maximal Kaplansky field, with its residue field relatively algebraically closed in that of $L$.

These results are a nice complement to the theory of tame fields as developed in [6]. The proofs of the above results will be given in Section 3, along with some more facts about algebraically maximal Kaplansky fields.

\section{Preliminaries}

We start with the following well known fact:

Lemma 2.1. If $(L, v)$ is henselian and $K$ a relatively algebraically closed subfield of $L$, then also $(K, v)$ is henselian.

Proof. Assume that $f$ is a polynomial with coefficients in the valuation ring of $(K, v)$ which satisfies the conditions of Hensel's Lemma. Since the valuation ring of $(K, v)$ is contained in that of $(L, v)$ and $(L, v)$ 
is henselian, $f$ admits a root $a$ in $L$ which satisfies the assertions of Hensel's Lemma. Being a root of $f, a$ is algebraic over $K$ and since $K$ is relatively algebraically closed in $L$, we have that $a \in K$.

Take a finite extension $(L \mid K, v)$ of valued fields. The Lemma of Ostrowski says that whenever the extension of $v$ from $K$ to $L$ is unique, then

$$
[L: K]=p^{\nu} \cdot(v L: v K) \cdot[L v: K v] \quad \text { with } \nu \geq 0
$$

where $p$ is the characteristic exponent of $L v$, that is, $p=$ char $L v$ if this is positive, and $p=1$ otherwise. For the proof, see [10, Theoreme 2, p. 236]) or [12, Corollary to Theorem 25, p. 78]). If $p^{\nu}=1$, then we say that the extension $(L \mid K, v)$ is defectless. Note that $(L \mid K, v)$ is always defectless if char $K v=0$. We call a henselian field a defectless field if all of its finite extensions are defectless. Each valued field of residue characteristic 0 is a defectless field.

An algebraic extension $(L \mid K, v)$ of henselian fields is called tame if every finite subextension $E \mid K$ of $L \mid K$ satisfies the following conditions: (TE1) if char $K v=p>0$, then the ramification index $(v E: v K)$ is prime to $p$,

(TE2) the residue field extension $E v \mid K v$ is separable, (TE3) the extension $(E \mid K, v)$ is defectless.

A tame valued field (in short, tame field) is a henselian field for which all algebraic extensions are tame. From the definition of a tame extension it follows that $(K, v)$ is a tame field if and only if it is a defectless field satisfying conditions (K1) and (K2). It is an easy observation that every extension of a defectless field $(K, v)$ satisfying conditions (K1) and (K2) must be tame and so $(K, v)$ must be a tame field. The converse can be derived from the arguments in the second part of the proof of Proposition 3.1 below.

By (K1) and (K2), the perfect hull of a tame field is an immediate extension, and by (TF3), this extension must be trivial. This shows that every tame field is perfect.

If char $K v=0$, then conditions (TE1) and (TE2) are void, and every finite extension of $(K, v)$ is defectless. Hence very algebraic extension of a henselian field of residue characteristic 0 is a tame extension, and every henselian field of residue characteristic 0 is a tame field.

Lemma 2.2. Every algebraically maximal valued field satisfying conditions (K1) and (K2), and in particular every algebraically maximal Kaplansky field, is a tame field and hence a perfect and defectless field. 
Proof. This follows from [6, Theorem 3.2] and the facts about tame fields we have mentioned above.

The converse of this lemma does not hold since for a tame field it is admissible that its residue field has finite separable extensions with degree divisible by $p$.

Take a valued field $(K, v)$, fix an extension of $v$ to $K^{\text {sep }}$ and call it again $v$. The fixed field of the closed subgroup

$$
G^{r}:=\left\{\sigma \in \operatorname{Gal}\left(K^{\mathrm{sep}} \mid K\right) \mid v(\sigma a-a)>v a \text { for all } a \in \mathcal{O}_{K^{\text {sep }}} \backslash\{0\}\right\}
$$

of $\operatorname{Gal}\left(K^{\mathrm{sep}} \mid K\right)$ (cf. [1, Corollary (20.6)]) is called the absolute ramification field of $(K, v)$. For the following fact, see [1, (20.15 b)].

Lemma 2.3. Take a henselian field $(K, v)$ and denote by $Z$ the absolute ramification field of $(K, v)$. If $\left(K^{\prime}, v\right)$ is an extension of $(K, v)$ inside of $Z$, then $Z$ is also the absolute ramification field of $\left(K^{\prime}, v\right)$.

The next result follows from [1, Theorem (22.7)] (see also [7, Proposition 4.1]).

Proposition 2.4. The absolute ramification field of $(K, v)$ is the maximal tame extension of $(K, v)$.

An algebraic extension of a henselian field is called purely wild if it is linearly disjoint to the absolute ramification field and thus to every tame extension. In [7] it was shown that maximal purely wild extensions are field complements to the absolute ramification field. In the case of Kaplansky fields, they are at the same time maximal immediate algebraic extensions, and they are unique up to isomorphism. See the cited paper for details.

Finally, we will need the following results about the absolute ramification field.

Lemma 2.5. Take an algebraic extension $(L \mid K, v)$ of henselian fields. Denote by $Z$ the absolute ramification field of $(K, v)$. Then $(Z \cap L, v)$ is a tame extension of $(K, v)$ maximal with respect to being contained in $L$, and $(L, v)$ is a purely wild extension of $(Z \cap L, v)$.

Proof. By Proposition 2.4, $(Z \cap L, v)$ is a tame extension of $(K, v)$. Assume that $(E, v)$ is a tame extension of $(K, v)$ inside of $(L, v)$ which is an extension of $(Z \cap L, v)$. Then $(E, v)$ is also a tame extension of $(Z \cap L, v)$. By Lemma $2.3, Z$ is also the absolute ramification field of $(Z \cap L, v)$. Thus by Proposition 2.4, $Z$ is the maximal tame extension of $(Z \cap L, v)$. Hence $E \subseteq Z$ and therefore, $E=Z \cap L$, which proves 
that $(Z \cap L, v)$ is a tame extension of $(K, v)$ maximal with respect to being contained in $L$.

Now we wish to prove that $(L \mid Z \cap L, v)$ is a purely wild extension. Since $Z$ is the absolute ramification field of $(Z \cap L, v)$, it suffices to show that $L \mid Z \cap L$ is linearly disjoint from $Z \mid Z \cap L$. But this follows from the fact that according to [2, Theorem 5.3.3 (2)], $Z \mid K$ is a Galois extension.

\section{Results On ALGEBRAICALly MAXIMAL KAPLANSKY FIELDS}

We start with the following characterization of algebraically maximal Kaplansky fields:

Proposition 3.1. A valued field $(K, v)$ is an algebraically maximal Kaplansky field if and only if it is henselian and does not admit any finite extension of degree divisible by $p$.

Proof. If char $K v=0$, then the assertion is trivial since in this case, $(K, v)$ is an algebraically maximal Kaplansky field if and only if it is henselian, and 0 does not divide the degree of any finite extension.

Now we consider the case of char $K v=p>0$. Assume first that $(K, v)$ is an algebraically maximal Kaplansky field. Then in particular, it is henselian. By Lemma 2.2, it is a defectless field, that is, every finite extension $\left(K^{\prime} \mid K, v\right)$ satisfies

$$
\left[K^{\prime}: K\right]=\left(v K^{\prime}: v K\right) \cdot\left[K^{\prime} v: K v\right] .
$$

Since $(K, v)$ satisfies $(\mathrm{K} 1),\left(v K^{\prime}: v K\right)$ is not divisible by $p$. Because of $(\mathrm{K} 2)$ and $(\mathrm{K} 3)$, the same holds for $\left[K^{\prime} v: K v\right]$. Hence also $\left[K^{\prime}: K\right]$ is not divisible by $p$.

Now assume that $(K, v)$ is henselian and does not admit any finite extension of degree divisible by $p$. By the Lemma of Ostrowski, every finite extension $\left(K^{\prime} \mid K, v\right)$ satisfies

$$
\left[K^{\prime}: K\right]=p^{\nu} \cdot\left(v K^{\prime}: v K\right) \cdot\left[K^{\prime} v: K v\right]
$$

with $\nu \geq 0$. Since $\left[K^{\prime}: K\right]$ is not divisible by $p$, it follows that $\nu=0$. This implies that $(K, v)$ is a defectless field and thus also an algebraically maximal field. Further, if there were an element $\alpha \in v K$ not divisible by $p$, then adjoining to $K$ the $p$-th root of any element of $K$ having value $\alpha$ would generate an extension of $K$ of degree $p$. This shows that $v K$ must be $p$-divisible, i.e., $(K, v)$ satisfies (K1). Finally, if there were a finite extension of $K v$ with a degree divisible by $p$, then it could be lifted to an extension of $K$ of the same degree, which should not exist. This shows that $K v$ does not admit any finite extension of degree divisible by $p$, so $(K, v)$ satisfies (K2) and (K3). 
Proof of Proposition 1.2. The first assertion is trivial if char $K v=0$ since a relatively algebraically closed subfield of a henselian field is again henselian. Suppose that $K v$ is not relatively algebraically closed in $L v$. Then there exists some $\bar{a} \in L v \backslash K v$ separable-algebraic over $K v$. Lift its minimal polynomial $\bar{f}$ over $K v$ up to a polynomial $f$ over $K$ of the same degree. Since $\bar{a}$ is a simple root of $\bar{f}$, Hensel's Lemma yields the existence of a root $a \in L$ of $f$ with $a v=\bar{a}$. Then $a$ is algebraic over, but not in $K$, which contradicts our assumption that $K$ is relatively algebraically closed in $L$. Hence $K v$ is relatively algebraically closed in $L v$.

Let us now assume that char $K v=p>0$. Then we can use the assertion of Proposition [3.1. Since $L$ is perfect by Lemma 2.2, the same holds for its relatively algebraically closed subfield $K$. Since the algebraically maximal field $(L, v)$ is henselian, the same holds for $(K, v)$ by Lemma 2.1 .

Take any finite extension $K^{\prime} \mid K$. Since $K$ is perfect and relatively algebraically closed in $L, K^{\prime} \mid K$ is linearly disjoint from $L \mid K$. Therefore, $\left[K^{\prime}: K\right]=\left[L . K^{\prime}: L\right]$, which is not divisible by $p$. In view of Proposition 3.1, this proves that $(K, v)$ is an algebraically maximal Kaplansky field. In particular, $K v$ is perfect. As in the case of char $K v=0$ it is now shown that $K v$ is relatively algebraically closed in $L v$.

Proposition 3.2. Take an algebraically maximal Kaplansky field $(L, v)$ and a henselian subfield $K$ such that $L \mid K$ is algebraic. Denote the absolute ramification field of $(K, v)$ by $Z$ and set $Z_{0}:=Z \cap L$. Then the following assertions hold:

a) $\left(Z_{0}, v\right)$ is a tame extension of $(K, v)$ maximal with respect to being contained in $L$,

b) $(L, v)$ is a maximal purely wild extension of $\left(Z_{0}, v\right)$,

c) (K3) also holds for $\left(Z_{0}, v\right)$.

Proof. The assertions are trivial if char $K v=0$ (in which case $Z_{0}=L$ ), so let us assume again that char $K v=p>0$.

It follows from Lemma 2.5 that $\left(Z_{0}, v\right)$ is a tame extension of $(K, v)$ maximal with respect to being contained in $L$, and that $\left(L \mid Z_{0}, v\right)$ is purely wild. In fact, $(L, v)$ is a maximal purely wild extension of $\left(Z_{0}, v\right)$ since by Lemma 2.2 it is a tame field. Since $\left(L \mid Z_{0}, v\right)$ is purely wild, the extension of the respective residue fields is purely inseparable. As $L$ satisfies (K3), the same is consequently true for $Z_{0}$.

Proposition 3.3. Take an algebraically maximal Kaplansky field $(L, v)$ and a subfield $K$ such that $L \mid K$ is algebraic. Then $(L, v)$ contains:

a) a maximal immediate algebraic extension of $(K, v)$, 
b) a henselization $\left(K^{h}, v\right)$ of $(K, v)$ and a maximal purely wild extension of $\left(K^{h}, v\right)$.

Proof. As before, the assertions are trivial if char $K v=0$, so let us assume again that char $K v=p>0$. Since the algebraically maximal field $(L, v)$ is henselian, it contains a henselization $K^{h}$ of $(K, v)$, and this is an immediate algebraic extension of $(K, v)$. Thus we may from now on assume that $(K, v)$ itself is henselian.

For the proof of part a), we take $\left(K^{\prime}, v\right)$ to be an immediate extension of $(K, v)$, maximal with respect to being contained in $L$. Then $\left(K^{\prime}, v\right)$ is henselian since it is an algebraic extension of the henselian field $(K, v)$. We set $Z_{0}:=Z \cap L$, where $Z$ is the absolute ramification field of $\left(K^{\prime}, v\right)$. Suppose that $\left(K^{\prime}, v\right)$ is not algebraically maximal. Then there exists a nontrivial immediate algebraic extension $\left(K^{\prime}(a) \mid K^{\prime}, v\right)$. Since it is purely wild, it is linearly disjoint from the tame extensions $Z \mid K^{\prime}$ and $Z_{0} \mid K^{\prime}$, and it follows that $Z_{0}(a) \mid Z_{0}$ is also linearly disjoint from $Z \mid Z_{0}$. By Lemma 2.3, $Z$ is also the absolute ramification field of $Z_{0}$, so this shows that $\left(Z_{0}(a) \mid Z_{0}, v\right)$ is purely wild. Since by Proposition $3.2, Z_{0}$ satisfies (K3), all maximal purely wild extensions of $\left(Z_{0}, v\right)$ are isomorphic over $Z_{0}$ by Proposition 3.2 of [7]. As $(L, v)$ is a maximal purely wild extension of $\left(Z_{0}, v\right)$ by Proposition $\left[3.2\right.$, it follows that $Z_{0}(a)$ admits an embedding in $L$ over $Z_{0}$. Hence there is some $a^{\prime} \in L$ such that $Z_{0}(a)$ and $Z_{0}\left(a^{\prime}\right)$ are isomorphic over $Z_{0}$. This isomorphism induces an isomorphism $K^{\prime}(a) \simeq K^{\prime}\left(a^{\prime}\right)$ over $K^{\prime}$. Since $\left(K^{\prime}, v\right)$ is henselian, this isomorphism preserves the valuation, so $\left(K^{\prime}\left(a^{\prime}\right) \mid K^{\prime}, v\right)$ is a nontrivial immediate algebraic extension contained in $L$. As this contradicts the maximality of $\left(K^{\prime}, v\right)$, we find that $\left(K^{\prime}, v\right)$ must be algebraically maximal.

In order to prove part b), take $\left(K^{\prime}, v\right)$ to be a purely wild extension of $K$, maximal with respect to being contained in $K$. Again, $\left(K^{\prime}, v\right)$ is henselian. By the same proof as before, just replacing "immediate" by "purely wild", one shows that $\left(K^{\prime}, v\right)$ is a maximal purely wild extension of $(K, v)$.

Proof of Theorem 1.1. In order to prove the first part of the theorem, we use Proposition 1.2 to replace $L$ by the relative algebraic closure of $K$ in $L$. Then the assertions follow from Proposition 3.3.

For the proof of the second part of the theorem, assume that $(L, v)$ is maximal, and take an immediate extension $\left(K^{\prime}, v\right)$ of $(K, v)$, maximal with respect to being contained in $L$. By the first part of our theorem, the relative algebraic closure of $K^{\prime}$ in $L$ contains a maximal immediate algebraic extension of $\left(K^{\prime}, v\right)$; by the maximality of $K^{\prime}$, it must be 
equal to $K^{\prime}$. Hence $\left(K^{\prime}, v\right)$ does not admit any nontrivial immediate algebraic extensions.

Suppose that $\left(K^{\prime}, v\right)$ is not maximal. Then by [3, Theorem 4], $\left(K^{\prime}, v\right)$ admits a pseudo Cauchy sequence without a limit in $K^{\prime}$. This must be of transcendental type, because if it were of algebraic type, then by [3, Theorem 3], there would exist a nontrivial immediate algebraic extension of $\left(K^{\prime}, v\right)$, contradicting its algebraic maximality. Since the pseudo Cauchy sequence we took in $K^{\prime}$ is also a pseudo Cauchy sequence in $(L, v)$ and $(L, v)$ is maximal, [3, Theorem 4] shows the existence of a limit $a$ in $L$. It follows from [3, Theorem 2] that $\left(K^{\prime}(a) \mid K^{\prime}, v\right)$ is an immediate transcendental extension. As it is contained in $L$, this contradicts the maximality of $\left(K^{\prime}, v\right)$, We have now proved that $\left(K^{\prime}, v\right)$, is a maximal immediate extension of $(K, v)$.

\section{REFERENCES}

[1] Endler, O.: Valuation theory, Springer, Berlin (1972)

[2] Engler, A.J. - Prestel, A.: Valued fields, Springer Monographs in Mathematics. Springer-Verlag, Berlin, 2005

[3] I. Kaplansky, Maximal fields with valuations I, Duke Math. Journ. 9 (1942), 303-321

[4] F.-V. Kuhlmann, Additive Polynomials and Their Role in the Model Theory of Valued Fields, Proceedings of the Workshop and Conference on Logic, Algebra, and Arithmetic, held October 18-22, 2003. Lecture Notes in Logic 26 (2006), 160-203

[5] F.-V. Kuhlmann, Approximation of elements in henselizations, manuscripta math. 136 (2011), 461-474

[6] F.-V. Kuhlmann, The algebra and model theory of tame valued fields, J. reine angew. Math. 719 (2016), 1-43

[7] F.-V. Kuhlmann, M. Pank, and P. Roquette, Immediate and purely wild extensions of valued fields, manuscripta math. 55 (1986), 39-67

[8] Prieß-Crampe, S.: Der Banachsche Fixpunktsatz für ultrametrische Räume, Results in Mathematics 18 (1990), 178-186

[9] Prieß-Crampe, S.: Reaching fixed points as limits in subspaces, to appear in J. Geometry

[10] Ribenboim, P.: Théorie des valuations. Les Presses de l'Université de Montréal, Montréal, 2nd ed. (1968)

[11] G. Whaples, Galois cohomology of additive polynomial and $n$-th power mappings of fields, Duke Math. J. 24 (1957), 143-150

[12] Zariski, O. - Samuel, P.: Commutative Algebra, Vol. II, New York-HeidelbergBerlin (1960)

Institute of Mathematics, University of Szczecin, Ul. WielKopolska 15, 70-451 Szczecin, Poland

E-mail address: fvk@math.us.edu.pl 